\title{
EMERGENCY DEPARTMENT PHARMACIST PRACTITIONERS: A NEW ROLE IN THE NHS
}

D. Greenwood, M. Tully, S. Martin, D. Steinke. Division of Pharmacy and Optometry, University of Manchester, UK

E-mail address: daniel.greenwood@manchester.ac.uk (D. Greenwood).

Background: Due to a shortage of emergency department (ED) doctors and nurses, hospitals have started to employ pharmacists with additional clinical training to help deliver services. Known as Emergency Department Pharmacist Practitioners (EDPPs), this new role has pharmacists providing hands-on care to patients in an emergency care setting. This study aimed to describe this new pharmacy service.

Methods: A self-administered survey method was employed. Over 10 work days, EDPPs reported their contribution to the care of individual ED patients via a purpose developed iPad questionnaire completed at the time of care. Structured around the patient management process, EDPPs reported both traditional clinical pharmacy activities (e.g. the clinical check of prescriptions) and activities associated with additional clinical training (e.g. clinical examinations).

Results: Twenty EDPPs from 15 UK hospitals were recruited, who each reported their care contributions for 3 to 10 days. Of 682 patients cared for, $4.8 \%(n=33)$ were of blue triage category (least urgent) and $4.1 \%(n=28)$ red (immediate need). Red triage category presenting complaints included myocardial infarction (8, 28.6\%) and shortness of breath $(4,14.3 \%)$. Overall, $53.2 \%$ of patients ( $n=363$ ) were admitted to hospital and $39.7 \%(n=271)$ were discharged home. Eleven EDPPs took on the role of main care provider for at least some of their patients. Thirteen EDPPs performed diagnostic clinical examinations (e.g. cardiovascular and neurology) for, on average, $62.8 \%$ of patients seen. Other services included prescribing medication to be given in the ED (17/20,44.4\%); and checking prescriptions for clinical appropriateness (14/20, 52.0\%).

Conclusions: EDPP services consist of hands-on patient care as well as traditional clinical pharmacy activities. This included being main care provider, normally the professional jurisdiction of doctors/nurse practitioners. Further analysis will explore the different roles and work patterns of EDPPs collectively and individually to define this new role. 